\title{
A Neural Network Based Approach for Exhibition Evaluation in Modern Science and Technology Museums
}

\author{
Qiusha Min, Yating Chen, Zhifeng Wang, Dan Xia, Mingzhang Zuo, and Junming Ye
}

\begin{abstract}
The Modern Science and Technology Museum is an important place for popularizing scientific knowledge and improving the scientific quality of the public, playing an important role in promoting scientific and technological progress. This paper presents a quantified evaluation method for exhibits in Science and Technology Museums. This method establishes a three-level index system and trains the weight of each index in the index system through neural network to evaluate exhibits quantitatively. Compared with traditional evaluation methods, this method can more comprehensively and scientifically evaluate the display effect of exhibits. Through this evaluation index system, administrator of Science and Technology Museum can know what aspects of the exhibit need to be improved. In response to these aspects, the exhibit is modified to improve its display effect.
\end{abstract}

Index Terms-Modern science and technology museum, exhibits, evaluation, neural network.

\section{INTRODUCTION}

Interactive experience and dynamic display exhibits are the main display carrier of the Modern Science and Technology Museum. The Modern Science and Technology Museum is open to the public throughout the year and is a public welfare science popularization venue that is not for profit [1]. Its main function is to popularize scientific and technologic knowledge to the public, disseminate scientific ideas and scientific methods and provide services of improving scientific and cultural literacy of the public, especially young people. Science and Technology Museum is an important place for popular science, shouldering the burden of carrying out science education and improving national scientific quality [2]. It is currently showing a booming momentum.

The typical exhibition forms of Modern Science and Technology Museums include permanent exhibitions and temporary exhibitions. Although these two types of exhibitions have obvious differences in exhibition time, contents and expressions, they are inseparable from the "exhibits" element. During the exhibition, it is the exhibits

Manuscript received January 3, 2018; revised September 12, 2018. This work was supported by National Key Technology Research and Development Program of the Ministry of Science and Technology of China (No.2015BAK33B02), the MOE (Ministry of Education in China) Project of Humanities and Social Sciences (No.17YJC880081 and 15YJA880095), and the self-determined research funds of CCNU from the colleges' basic research and operation of MOE (No. CCNU17QN0015).

The authors are with Central China Normal University, Hubei, China (e-mail: qiusham@mail.ccnu.edu.cn,_ytchen@mails.ccnu.edu.cn, zfwang@mail.ccnu.edu.cn, mzzuo@mail.ccnu.edu.cn, jmye@mail.ccnu.edu.cn). displayed in the Science and Technology Museum that connects the general public with Science and Technology Museums [2]. Exhibits are the foundation of Science and Technology Museums and the main carriers of Science and Technology Museums to implement education. However, staff of Science and Technology Museums do not know how well these exhibits are displayed. Therefore, how to evaluate the effect of Science and Technology Museums comprehensively and scientifically through specific evaluation indicators has become an important part of the construction of Modern Science and Technology Museums [3]. In order to evaluate the display effect of the exhibits in the Science and Technology Museum, a set of effective evaluation system should be established firstly, through which experts evaluate each evaluation index. Then the display effect of this exhibit can be calculated according to the score of each index and its weight. This paper established a comprehensive evaluation system, and also introduced how to determine the weight of each index in this evaluation system through neural network. Finally, the display effect of a certain exhibit in the Science and Technology Museum can be calculated according to the formula.

\section{ESTABLISHMENT OF THE EVALUATION INDEX SySTEM}

The index system for evaluating the display effect of exhibits in Science and Technology Museums consists of three levels. The first level consists of seven first-level indexes: scientific, interactive, interesting, safe, experiential, harmonious and economical. The second level is the subdivision of seven first-level indexes, the first-level indexes are divided into several second-level indexes; the third level is composed of quantifiable third-level indexes belonging to the second-level indexes respectively.

\section{A. Scientificity}

The basic characteristic that exhibits must have is scientificity [4]. If scientific errors occur, it will not only violate the original intention of popularizing scientific knowledge but will also mislead visitors. The cooperation between Science and Technology Museums and schools has been an emerging topic in the education community in recent years. To evaluate the scientificity of the exhibit, Science and Technology Museums can design some educational exhibition activities related to school curriculum to meet the needs of students. After the relevant courses in the school help students understand relevant scientific knowledge, students can visit the Science and Technology Museum to see the corresponding exhibits and start exploratory learning. It will help cultivate students' scientific and cultural literacy. 
Finally, staff of the Science and Technology Museum can test the results of these activities and analyze the data to evaluate the scientificity of the exhibit.

As shown in Fig. 1, the scientificity can be subdivided into two second-level indexes: scientificity in principle and scientificity in design. The scientificity in principle includes: whether the display process of the exhibits reflects the correct scientific and technological principles, whether the display effect of exhibits correctly reflects the role of science and technology in promoting human progress and social development. The scientificity in design includes: whether the structural design of the exhibits meets the needs of human real life, whether the formal design of the exhibition meets the content of the exhibits.

\section{B. Interactivity}

Interactivity is one of the most vital features of the exhibits in Science and Technology Museum. The uniqueness of Science and Technology Museums differs from traditional museums is that it emphasizes the interactivity of exhibits [5]. It emphasizes finding problems, thinking about problems, and solving problems in interactions, so as to gain the joy of experience and enlightenment of thinking. The exhibits in Science and Technology Museums should inspire and satisfy the desire of the audience to actively study and explore and let them become the active explorers of knowledge [6].

Through some behaviors such as the interaction between the audience and the exhibit or taking pictures, the interactivity of the exhibit can be measured. The RFID identification technology can obtain the location information and the dwell time of the audience area through the devices carried by the audience to evaluate the interactivity of the exhibits. It can cover the recognition network in the exhibition area by wearing electronic tags (RFID cards, etc.) with identification functions on the audience. The data acquisition system adopted by the RFID identification technology performs real-time detection through a background program and collects the information of the viewer's action route and the time spent in the area. The collected information is combined with the feedback data that audiences operate exhibits in the real venue. Through data mining and comprehensive analysis techniques, the audience's visit behavior was comprehensively analyzed and the interactivity of the exhibits can be measured.

As shown in Fig. 2, the interactivity is divided into some second-level evaluation indexes: the degree of interaction, other sensory participation and the convenience of the operation. The degree of interaction includes: whether the interaction time between exhibits and audiences is more than 3 minutes, whether the number of interactions is more than one. Other sensory participation includes: whether the exhibit needs the participation of audience's eyes in the exhibition process; whether it needs audience's ear for interaction; whether it requires the participation of the nose and whether it needs the participation of the mouth. The convenience of the operation includes: whether the operation steps of the exhibit are within 5 steps; whether the audience can start operating the exhibit within 10 seconds; whether the requirements of the exhibit on the audience during the operation are within 2 or less.

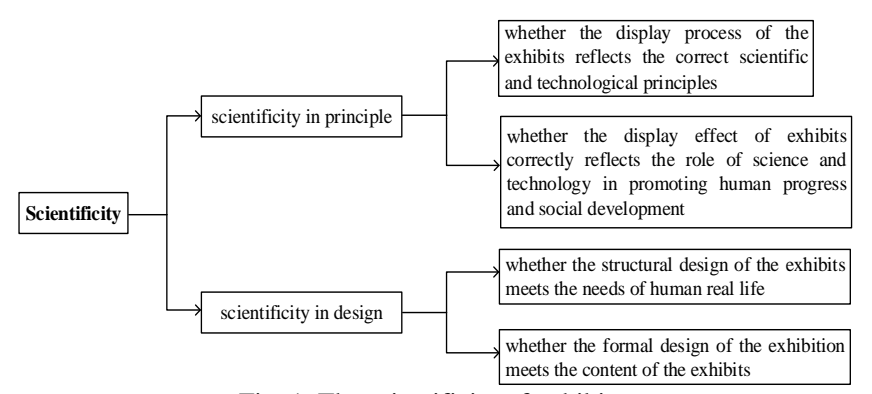

Fig. 1. The scientificity of exhibits.

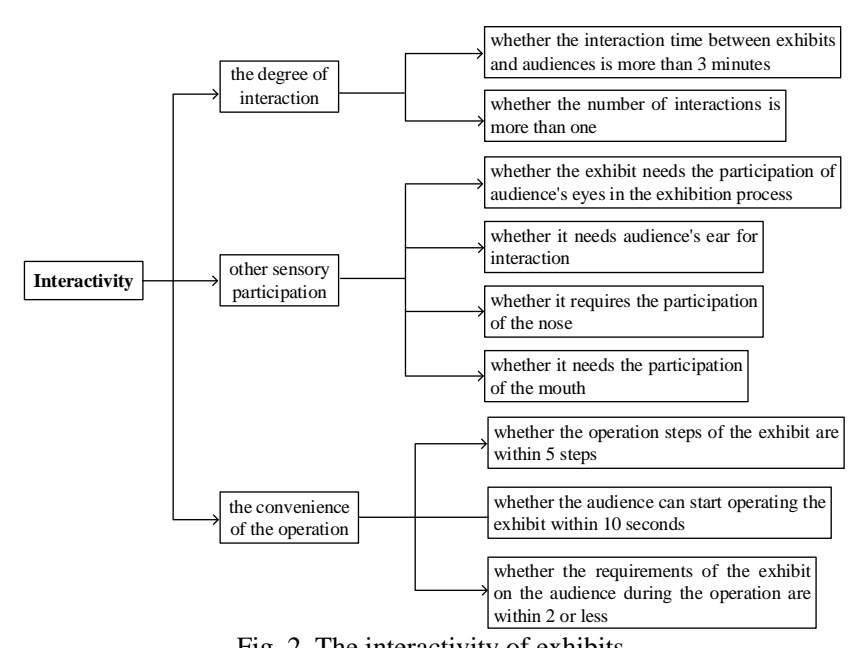

Fig. 2. The interactivity of exhibits.

\section{Fun}

The route for the audience to visit the Science and Technology Museum is arbitrary, and the visit process is full of relaxed, free, and active atmosphere. Therefore, in order to achieve the purpose of popularizing scientific knowledge to the audience, it is necessary to increase the interest of exhibits. Victor Daniloff believes that successful exhibits must enable the audience to generate and maintain interest.

Through the three-dimensional face scanning technology to scan the audience's facial expressions during the visit, the fun of exhibits can be measured. To achieve three-dimensional scanning, a database needs to be created firstly. A powerful database is an information database for face recognition. When the audience stands in front of the 3D face scanning cabinet and activates the scan button, the system will instantly extract the feature points of the audience's face. At the same time, the system will automatically mark the desired feature points on the face of the audience, such as the outer corner of the eye, the inner corner of the eye, the point between the eyebrows, spinal points, gnathion and mouth corners, etc., recording audience's facial expressions of joy and grief as they manipulate the exhibits. The more pleasing the expression gets, the more interesting the exhibit is.

As shown in Fig. 3, the fun of the exhibits is divided into two second-level evaluation indexes: the degree of interest and the degree of participation. The degree of interest includes: whether the audience will take pictures or videos on the exhibit; whether the audience is happy and their voices are excited when visiting the exhibit. The degree of participation includes: whether the audience residing time is more than 3 minutes when visiting the exhibit; whether the number of times the audience repeatedly experiences the 
exhibit is more than 2 times; whether the number of participants of the exhibit accounts for more than $30 \%$ of the total number of people visiting the Science and Technology Museums.

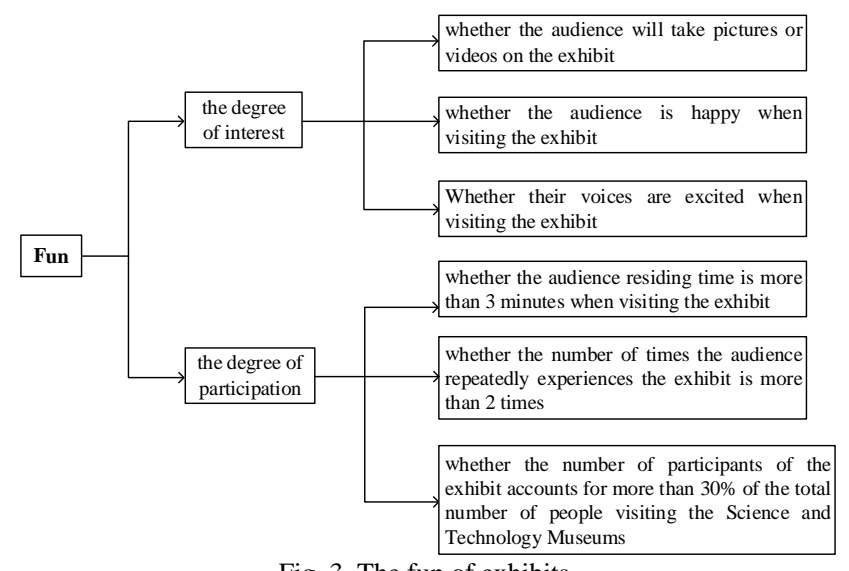

Fig. 3. The fun of exhibits.

\section{Safety}

Modern Science and Technology Museums increasingly use interactive display technology in exhibits, and electromechanical-driven exhibits are everywhere. In comparison, the security issues of Science and Technology Museums are much more acute than other museums or exhibition halls [5]. The safety of exhibits includes personal safety of audience and safety of instruments. In the design and manufacture of exhibits, safety is a top priority. For exhibits involving high temperature, high pressure, strong electricity and exhibits that are flammable, explosive or poisonous, the staff must make as many predictions of potential safety hazards as possible, take appropriate protective measures and resolutely eliminate the existence of hidden accidents. When there is a conflict between safety, Scientificity and interactivity, the staff must absolutely obey the principle of safety first.

In addition, there are many interactive exhibition items in Science and Technology Museums are large-scale sports projects, which are prone to potential safety hazards. For example, when the audience rotates on the three-dimensional scroll ring, they will feel awkwardly turning. During the moonwalk, the weight of the audience should be controlled. Otherwise, the following danger may occur: audience with a weight less than $45 \mathrm{~kg}$ may be hung up and cannot come down; if an audience whose weight is more than $80 \mathrm{~kg}$ is hung up, it may cause the rope to break. Therefore, these two types of people are forbidden to participate in moonwalk activities. During the exhibition, the Science and Technology Museum should remind the audience of safety in various forms, such as eye-catching sign boards.

As shown in Fig. 4, the safety of exhibits is divided into two second-level evaluation indexes: personal safety and instrument safety. Personal safety includes: whether the audience will be in danger when visiting the exhibit; whether the audience was informed about the relevant protection measures before they visited the exhibit. Instrument safety includes: durability, that is, whether the number of uses of this exhibit is greater than the average number of uses of exhibits in the Science and Technology Museums; the degree of oldness, that is, whether the ratio of the use life of this exhibit to their lifetime is less than 0.5 ; the maintenance rate, that is, whether the number of maintenances of these exhibits is less than or equal to the average number of maintenances of exhibits in the Science and Technology Museum.

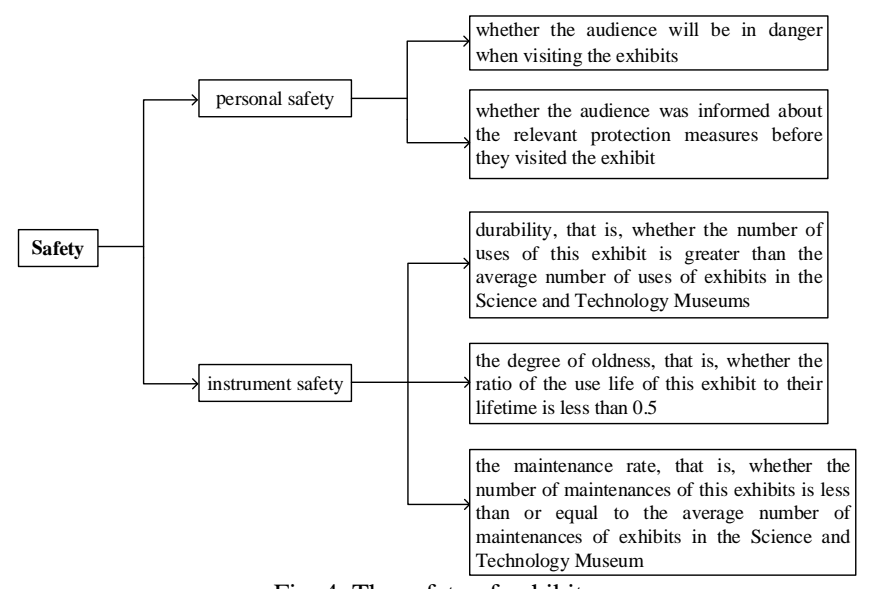

Fig. 4. The safety of exhibits.

\section{E. Experience}

As shown in Fig. 5, the experience of the exhibits is divided into two second-level evaluation indexes: the participation of the activities of exhibits and the simplicity of the operation of the exhibits. The participation of the activities of exhibits include: the type of participation when audiences experience the activities, that is, the exhibit show the technical principles to the audience alone, or audiences participate in the exhibition activities to control the exhibit for next operation; the degree of participation, that is, audiences' interaction with the exhibit when participating in activities of the exhibit is whether they experience the activities themselves or assist others in participating in the activities. The simplicity of the operation of the exhibits includes: the complexity of the operation, that is, whether the step that audiences operate the exhibit is more than 10 steps; the time taken to start the operation, that is, whether the time for audiences to start operating the exhibit is more than 10 seconds.

\section{F. Harmony}

If the overall appearance of the exhibit and the surrounding environment are harmonious and organically integrated, it will be easier to retain audiences. The appearance of the exhibit includes shape, color, man-machine relationship processing, and so on. Good handling of these aspects will give people a sense of beauty, attract audience, and make people have the desire to participate and learn. If the exhibits are monotonous in color and sturdy in shape, despite their rich knowledge, it is difficult to stimulate audiences' desire to participate. Therefore, the modeling scale of the exhibits must conform to ergonomic requirements and should be easy for the audience to visit. The shape, color, decoration and texture of the exhibit should conform to the rules of visual communication and be universal to the audiences. The overall appearance of the exhibit should give the audience a sense of harmony. Attention should be paid to the simplicity and beauty of the exhibit, without excessive floral ornamentation. The surface treatment should avoid rough and 
simple, but also prevent excessively gorgeous or glare.

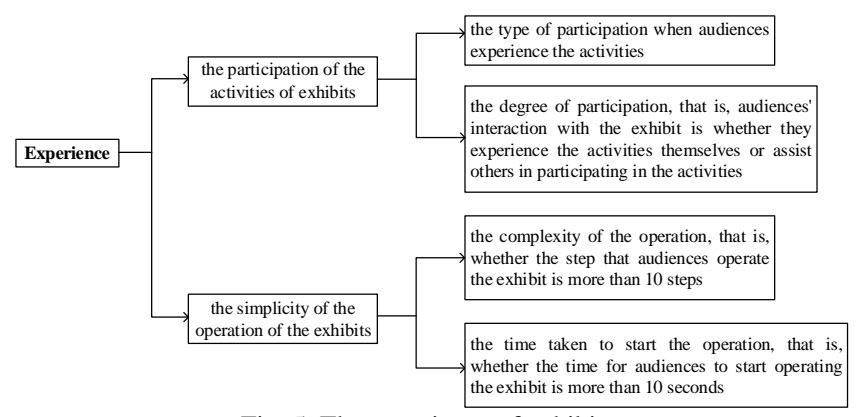

Fig. 5. The experience of exhibits.

The material of the exhibits can be seen as a combination of material and texture. It is a comprehensive sense of the various visual properties of the surface. These properties include surface texture, smoothness, transparency, reflectivity, luminosity, and more. The material should be unified with the light. The light of exhibits can be divided into direct and indirect light. The outstanding exhibits are the combination of two kinds of lights, which can show the effects that two kinds of lights do not have when they are used separately, emitting unique artistic rhyme. The entire atmosphere in the exhibition area is created by indirect light, which creates the atmosphere subtly and gives people a sense of harmony.

As shown in Fig. 6, the harmony of the exhibits is divided into two second-level evaluation indexes: the degree of harmony and the impact on audiences. The degree of harmony includes scientificity and experience. These two indexes are the first-level indexes mentioned above. The impact on the audience can be measured through questionnaires after the exhibition is over. For example, whether the halls, decorations, colors of the exhibit, lighting and temperature have a positive or negative impact on audiences' visit to the exhibit [7].

\section{G. Economy}

Economy means evaluating the educational role of exhibits from the perspective of investment efficiency [8]. From the perspective of economy, Science and Technology Museums should strive to increase the use rate of exhibits, highlight characteristics such as solidity, durability, repeated use, and multi-purpose use; try to do as little as possible one-time exhibits and reduce costs; try to avoid exhibits that are too expensive and reflect too limited scientific content to obtain the greatest educational effect with relatively small investment. The economy of the exhibit mainly depends on three aspects: 1) The actual accumulated running time of the exhibit, that is, the life of the exhibit. 2) The cost of the exhibit, including production costs, maintenance costs and operating costs. 3) The degree of influence of the exhibit on the audience.

The staff of the science and technology museum can connect local end equipment and the system in the museum through the IP network, so that the exhibits have good extendibility. Through the business intelligence platform to achieve a unified control interface, managers are provided with intuitive operating information and control. Therefore, they can control the business anytime anywhere through the TV, set-top box, mobile phone, PDA, laptops, etc., effectively improving the economy of exhibits.

As shown in Fig. 7, the economy of exhibits is divided into these second-level evaluation indexes: the cost of exhibits, the use of exhibits, the purpose of exhibits, and the reflection of scientific content. The cost of the exhibits includes: whether the design and production costs of the exhibit are higher than the average cost of exhibits in the Science and Technology Museum; whether the maintenance cost of the exhibit is higher than the average maintenance cost of the exhibits in the Science and Technology Museum. The use of exhibits includes: whether the time of use of the exhibit is greater than the average time of use of the exhibits in the Science and Technology Museum; whether the use of the exhibit will cause certain environmental problems or other problems. The purpose of exhibits includes: monomer usability and multi-purpose. Monomer usability refers to whether the exhibit can be used alone in real life. The multi-purpose of the exhibit refers to whether there are multiple functions of the exhibit in real life. The reflection of scientific content includes: whether the number of scientific principles reflected in the exhibit is more than one; whether the exhibit needs to consume energy such as water energy or electric energy when reflecting scientific content.

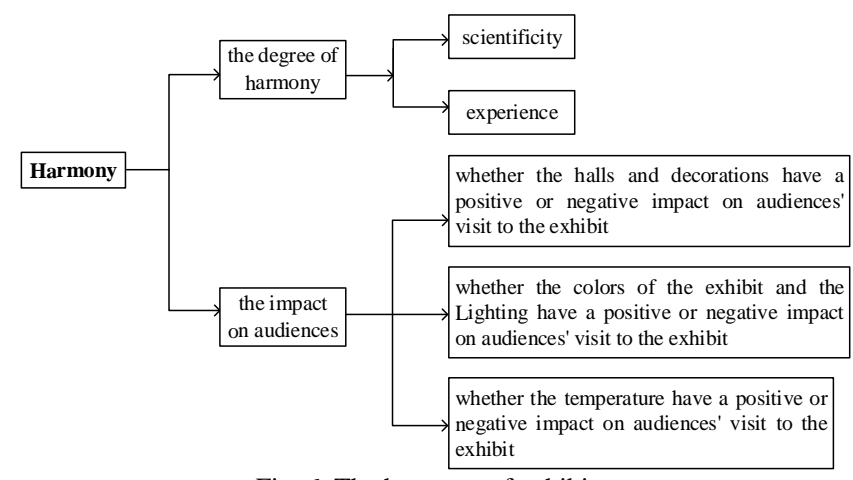

Fig. 6. The harmony of exhibits.

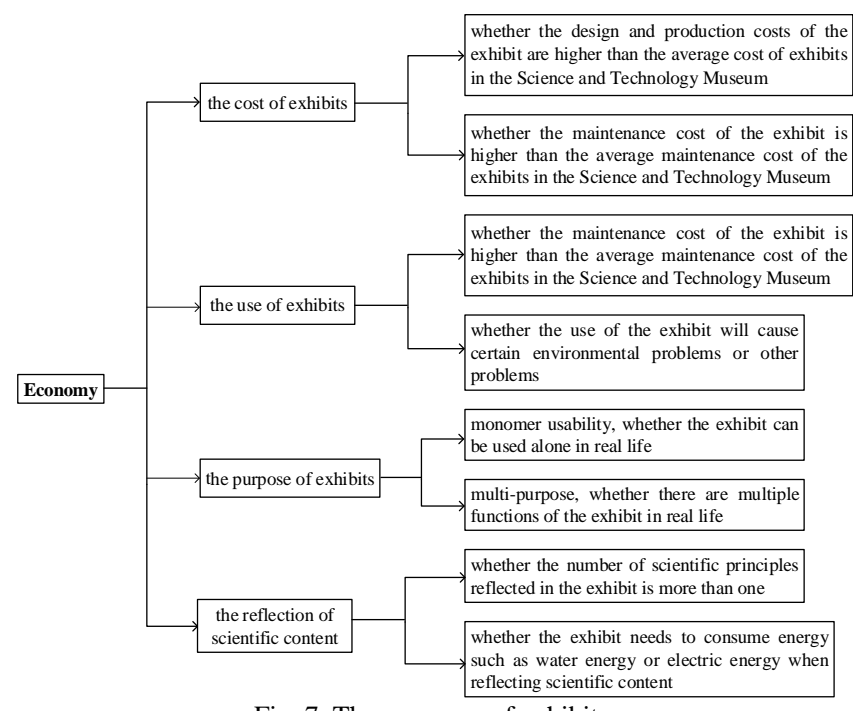

Fig. 7. The economy of exhibits.

\section{Determination OF THE WEIGHT OF EACH INDEX}

After the evaluation index system for exhibits is established, it is necessary to determine the weight of each index. In the assessment process, the importance of each indicator is different. Some indexes should occupy a larger 
weight, and some indexes' weight should be smaller. According to the evaluation index system of exhibits established above, this paper will use the neural network algorithm in machine learning and combine the expert's score on the indexes to determine the most appropriate weight for each index [9]. The unit of a neural network is a neuron, which are also called a perceptron [9]. The structure of a perceptron is shown in Fig. 8.

According to the evaluation index system established above, experts need to score each index. The total score is 5 . If the standard is reached, the corresponding score is given. If it is not reached, the score is 0 . The score of the three-level index is equivalent to the input $x i$ of the neural network, and the score of the first-level index is equivalent to the output $y$ of the neural network. The evaluation scores of experts on the exhibit are preserved as training samples for the neural network. For example, to evaluate the economy of exhibits, experts score the eight third-level indexes firstly. The scores of these eight three-level indexes are used as the input $x 1, x 2$, $x 3, x 4, x 5, x 6, x 7$ and $x 8$. In Fig. $8, w i(i=1,2, \ldots, 8)$ is the weight corresponding to the input $x i(\mathrm{i}=1,2, \ldots, 8)$ and $w 0$ can be regarded as the weight of the input $x b$ whose value is always 1 [9].

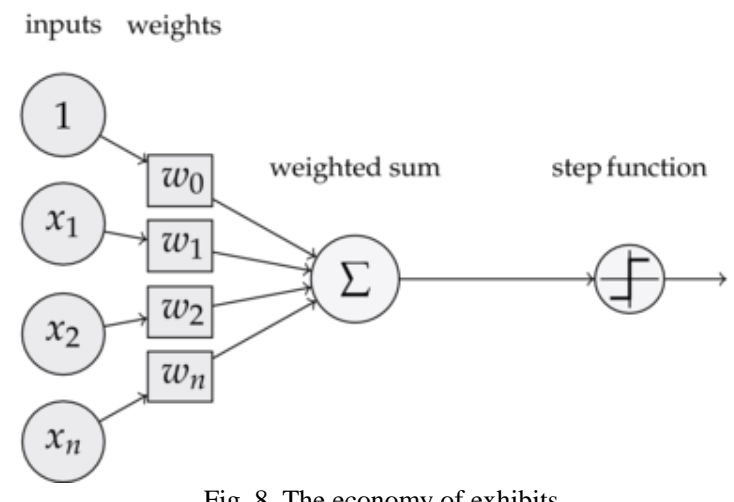

Fig. 8. The economy of exhibits.

To obtain the values of the weights and offset, the perceptron training algorithm will be used: first, the weight wi $(i=1,2, \ldots, 8)$ of each input and the offset $b$ (w0 in the figure) are initialized to 0 . Then the following perceptron rules (1) and (2) will be used to iteratively modify wi and $b$ until the training is completed [10].

$$
\begin{gathered}
w i \leftarrow w i+\triangle w i \\
b \leftarrow b+\triangle b
\end{gathered}
$$

where $\Delta w i$ and $\Delta b$ are calculated by (3) and (4):

$$
\begin{gathered}
\triangle w i=\eta(t-y) x i \\
\triangle b=\eta(t-y)
\end{gathered}
$$

$t$ is the actual value of the training sample, which is generally called label. And $y$ is the output value of the sensor, which is calculated according to formula (5). $\eta$ is a constant called the learning rate. Its role is to control the magnitude of each step adjustment.

$$
y=f(w * x+b)
$$

The neural network takes the score of three-level indexes of an exhibit from the training sample as the input vector $x$, the output $y$ is calculated using the formula (5). Then the calculated output $y$ is compared with the actual value of the first-level index of the exhibit. The weight $w$ is iteratively adjusted according to the perceptron rules (1) and (2) above. Each time a sample of the exhibits is processed, the weight of each index is adjusted. After multiple rounds of iterations (that is, the training data of all exhibits scores are processed repeatedly for multiple rounds), the weight of each index in the evaluation index system can be trained.

\section{APPLICATION}

With the help of the neural network to determine the weight of the evaluation indexes of the exhibits, the evaluation of the display effect of the exhibits in the Science and Technology Museums will be more accurate. The experts only need to score each measurable third-level index according to the scoring standard. The weight of each index is obtained through neural network training. Such an assessment result will be more accurate. The display effect evaluation system can provide reference for the Science and Technology Museums and related management work, promoting the development of the Science and Technology Museums better [11]. The staff of the Science and Technology Museum can use the display effect evaluation system to know which aspects of the exhibits have problems and adjust the factors for the lower evaluation of the exhibits [12]. If the score of interactivity is lower than other first-level indexes, the interactivity of the exhibit will be adjusted to further improve its display effect. In order to ensure the educational role of popular science education activities, the audience can learn some scientific and technological knowledge and principles through exhibits. It improves the public's scientific and cultural literacy and achieves the purpose of the Science and Technology Museums serving the public.

\section{CONCLUSION}

With the rapid development of the Science and Technology Museums, the evaluation of the display effect of exhibits will attract more and more attention. How to comprehensively and scientifically evaluate the display effect of exhibits of the Science and Technology Museums has become an urgent issue that needs to be solved in the development and management of Science and Technology Museums. This paper established a set of evaluation index system to evaluate the display effect of exhibits. At the same time, it introduced how to determine the weight of each index in this evaluation system through neural network. Finally, the display effect of a certain exhibit in the Science and Technology Museum can be evaluated according to the formula. It is hoped that this evaluation index system can 
provide reference for the Science and Technology Museums and related management work to better promote the development of the Science and Technology Museums.

\section{REFERENCES}

[1] S. Dong et al., "The development and evaluation of Chinese digital science and technology museum," Journal of Cultural Heritage, vol. 12, no. 1, pp. 111-115, 2011.

[2] A. Basso, F. Casarin, and S. Funari, "How well is the museum performing? A joint use of DEA and BSC to measure the performance of museums," Omega, 2017.

[3] H. Ding, Y. Sun, J. Liu, and M. Li, "Research on the validity of modern comprehensive evaluation methods," Journal of Convergence Information Technology, vol. 7, no. 22, pp. 118-127, 2012.

[4] D. L. Perry, "The museum impact and evaluation study: How visitors relate to science and technology museums," Visitor Studies, vol. 5, no. 1, pp. 254-259, 1992.

[5] A. Basso, and S. Funari, "Measuring the performance of museums: Classical and FDH DEA models," Rend.studi Econ.quant, pp. 1-16, 2004.

[6] A. Basso and S. Funari, "A quantitative approach to evaluate the relative efficiency of museums," Journal of Cultural Economics, vol. 28, no. 3, pp. 195-216, 2004.

[7] C. J. Maddison, A. Huang, I. Sutskever, and D. Silver, "Move evaluation in go using deep convolutional neural networks," Computer Science, 2014.

[8] M. J. D. Barrio and L. C. Herrero, "Evaluating the efficiency of museums using multiple outputs: evidence from a regional system of museums in Spain," International Journal of Cultural Policy, vol. 20, no. 2, pp. 221-238, 2014.

[9] O. Paulus, "Measuring museum performance: A Study of museums in France and the United States," International Journal of Arts Management, vol. 6, no. 1, pp. 50-63, 2003.

[10] A. Zorloni, "Designing a strategic framework to assess museum activities," International Journal of Arts Management, vol. 14, no. 2, pp.31-47, 2012.

[11] S. Birger et al., "Virtual museum system evaluation through user studies," Journal of Cultural Heritage, 26, 2017.

[12] R. S. Kaplan, "Strategic performance measurement and management in nonprofit organizations," Nonprofit Management \& Leadership, vol. 11 , no. 3, pp. 353-370, 2001.

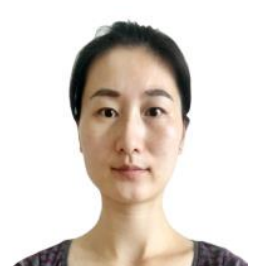

Qiusha Min received the B.S degree in educational technology from Jianghan University, Wuhan, China, in 2007, and the Ph.D. degree in engineering from Dublin City University, Dublin, Ireland, in 2013.

She is currently a lecturer in the School of Educational Information Technology, Central China Normal University. Her main research interests include web/mobile application development, multimedia systems, and educational technologies, focusing on blended learning approaches.

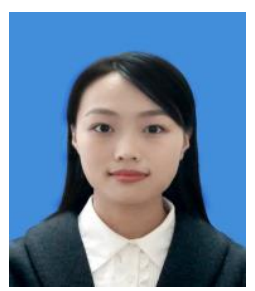

Yating Chen received the B.E degree from HuBei Normal University, HuangShi, China, in 2017.

She is currently a postgraduate in the School of Educational Information Technology, Central China Normal University. Her main areas of research are learning analytics and machine learning.

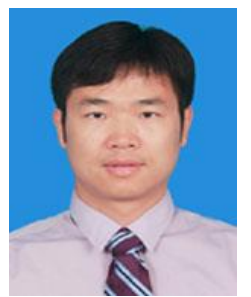

Zhifeng Wang received the B.S degree from China University of Geosciences, Wuhan, China, in 2008 , and the Ph.D. degree from South China University of Technology, Guangzhou, China, in 2013

$\mathrm{He}$ is currently an associate professor in the School of Educational Information Technology, Centra China Normal University. His main research interest include artificial intelligence, image and audio/video intelligent information processing, machine learning, human-computer interaction, virtual reality, game technology, data mining and multimedia forensics.

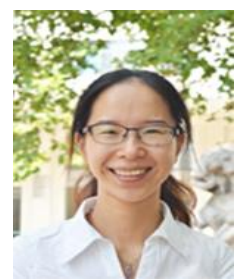

Dan Xia received the Ph.D. degree from Huazhong University of Science and Technology, Wuhan, China. She is currently a lecturer in the School of Educational Information Technology, Central China Normal University. Her main research interests include $3 \mathrm{D}$ reconstruction, image processing, computer vision and computer graphics.

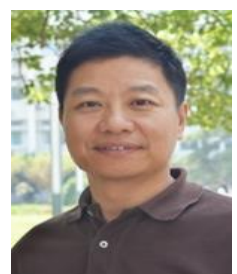

Mingzhang Zuo received the B.S degree in Electrochemistry Education from Central China Normal University, Wuhan, China, in 1991, the M.A degree in Educational Technology from Beijing Normal University in 1999, and the Ph.D. degree in Curriculum and Teaching Theory from Central China Normal University, Wuhan, China, in 2008.

$\mathrm{He}$ is currently a professor in the School of Educational Information Technology, Central China Normal University. His main research interests include educational technology theory and application, education informationization, and digital media.

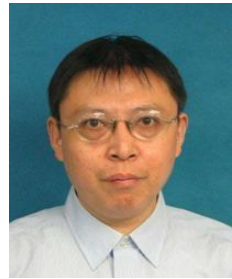

Junmin Ye is currently a professor in the School of Computer, Central China Normal University. His main research interests include educational big data and learning analysis, software analysis and verification and model-based software testing. 